\title{
Une approche diachronique des TMS : usage de données quantitatives dans une grande entreprise
}

A Diachronic Approach to MSD: Use of Quantitative Data in a Large Company Un enfoque diacrónico de los TME: uso de datos cuantitativos en una gran empresa

Céline Mardon, Willy Buchmann et Serge Volkoff

\section{Q OpenEdition}

\section{Journals}

Édition électronique

URL : http://journals.openedition.org/pistes/3530

DOI : 10.4000/pistes.3530

ISSN : 1481-9384

Éditeur

Les Amis de PISTES

Référence électronique

Céline Mardon, Willy Buchmann et Serge Volkoff, « Une approche diachronique des TMS : usage de données quantitatives dans une grande entreprise », Perspectives interdisciplinaires sur le travail et la santé [En ligne], 15-3 | 2013, mis en ligne le 01 décembre 2013, consulté le 30 avril 2019. URL : http:// journals.openedition.org/pistes/3530; DOI : 10.4000/pistes.3530

Ce document a été généré automatiquement le 30 avril 2019.

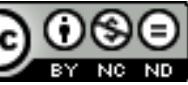

Pistes est mis à disposition selon les termes de la licence Creative Commons Attribution - Pas d'Utilisation Commerciale - Pas de Modification 4.0 International. 


\title{
Une approche diachronique des TMS : usage de données quantitatives dans une grande entreprise
}

\author{
A Diachronic Approach to MSD: Use of Quantitative Data in a Large Company \\ Un enfoque diacrónico de los TME: uso de datos cuantitativos en una gran \\ empresa
}

Céline Mardon, Willy Buchmann et Serge Volkoff

\section{NOTE DE L'ÉDITEUR}

Cet article a été originalement publié en portugais dans « Laboreal volume IX, no 1, 2013, pp 41-59. »

\section{Introduction}

Ces dernières années, de grands progrès ont été accomplis dans la compréhension des mécanismes d'apparition des douleurs articulaires et des troubles musculo-squelettiques (TMS) liés au travail, ainsi que dans leur prévention. Il apparaît cependant toujours justifié socialement et scientifiquement de mener des recherches sur le sujet afin de poursuivre cet effort de compréhension, même si, comme le notent Aptel et Vézina (2008), le progrès des connaissances n'implique pas forcément un progrès dans l'action :

«[...] Force est d'admettre que cela n'induit pas pour autant de volonté collective effective d'action à la hauteur de la réalité du risque. Plus encore, on assiste à un découplage entre le niveau de connaissance sur ce sujet et l'engagement des décideurs dans la prévention. Tout semble se passer comme si les TMS représentaient un handicap socialement accepté. » 
2 Dans ce contexte, la comptabilisation du nombre de personnes touchées par ces pathologies, comme plus largement par les maladies professionnelles (MP), représente un sujet délicat, tant elle manifeste des enjeux sociaux et économiques susceptibles d'influencer le fonctionnement des systèmes qui les gèrent. Le dénombrement des TMS n'échappe pas à ces enjeux, d'autant que ces pathologies constituent dans l'Union européenne la première cause de maladie professionnelle (Eurogip, 2007). Par ailleurs, toute comparaison européenne est relative et les conditions juridiques nationales de la réparation pèsent sur les statistiques de MP (id.). Pour ce qui concerne la France, en 2010, les MP relatives aux affections périarticulaires du membre supérieur représentaient près de $80 \%$ du total des MP reconnues. Mais de récentes études incitent à ne pas considérer le nombre de TMS reconnus comme une grandeur représentative de l'ampleur du phénomène (Ha et Roquelaure, 2010). Ces chiffres masquent l'histoire des salariés, des prémices de la douleur jusqu'au processus administratif de déclaration, voire de reconnaissance, de la maladie. Ils représentent un indicateur bien restrictif pour évaluer finement l'ampleur du phénomène TMS au vu des sous-déclarations (travailleurs dont l'état de santé ostéo-articulaire pourrait entrer dans le cadre réglementaire de réparation, mais qui n'engagent pas, pour diverses raisons, cette procédure administrative de déclaration en MP) et des «non-déclarations » (travailleurs présentant une pathologie ostéo-articulaire liée au travail mais qui ne rentre pas dans le cadre des tableaux de la CNAM) (Diricq, 2011). Il conviendrait donc de s'intéresser à d'autres indicateurs, si l'on entend décrire plus précisément l'épidémie de TMS à l'échelle de l'ensemble de la population active.

3 Selon le niveau hiérarchique - de l'opérateur atteint de douleurs articulaires à la direction d'entreprise en passant par le collectif de travail ou la hiérarchie de proximité les préoccupations liées à la gestion des TMS ne sont pas du même ordre. Malgré cela, tous s'accordent à dire que les TMS sont un problème coûteux, de différentes manières (Fauconnier et coll., 2005). Dans ce contexte, le vieillissement d'ensemble de la population active dans les pays industrialisés (Volkoff, 2012), avec l'impact incertain des politiques d'allongement de la vie professionnelle, les aspirations des salariés en mauvaise santé à un départ précoce en retraite, mais aussi côté travail, la persistance de contraintes physiques ou encore l'intensification du travail (Askenazy et coll., 2006) nous incitent à placer les conditions de travail et la santé aux fondements des débats scientifiques et sociaux sur ces sujets, et à adopter une lecture diachronique des faits étudiés, par une approche combinée des relations santé-travail, au fil des parcours professionnels.

4 Les théories de la santé et de la sécurité au travail, les apports de l'épidémiologie, les travaux de médecine du travail encouragent à insister sur l'intérêt de prendre en compte l'imbrication de plusieurs dimensions temporelles, de moyen et long termes (Plouvier, 2011 ; Aptel et Vézina, op. cit.).

5 Compléter l'analyse in situ des situations de travail par une approche diachronique constituerait donc un apport à la prévention (Cole et coll., 2002 ; Buchmann et coll., 2011). Les TMS sont issus de conditions d'exposition complexes dont les caractéristiques s'élaborent au fil des changements successifs (évolution des techniques de production, évolutions organisationnelles, ou encore évolutions des compétences, des collectifs de travail, etc.). Un regard croisant ces évolutions de type structurel avec l'arrivée de signes précurseurs sur la santé peut donner des clés nouvelles d'interprétation. Ces signes précurseurs, comme les douleurs considérées dans la suite du présent article, sont d'autant plus importants à prendre en compte que les TMS présentent des épisodes aigus 
pouvant devenir chroniques (Aptel et Vézina, op.cit.), et qu'il n'y a pas de décours temporel univoque d'un TMS (Aublet-Cuvelier et coll., 2006 ; Silverstein et coll., 2006). Il s'agit donc, par une approche compréhensive qui puisse prendre en compte le temps qui s'écoule à moyen et long termes (Berg et coll., 1988), de continuer à démêler l'écheveau des relations entre le travail et la santé.

Dans notre travail de recherche, articulant des approches qualitatives et quantitatives (Mergler, 1999) et divers niveaux (du micro sur le poste de travail au macro à l'échelle du groupe industriel), nous avons confronté différents points de vue disciplinaires en faisant dialoguer ergonomie, statistique, ou encore médecine du travail, avec pour fil conducteur la diachronie (id.). Cet article n'a pas pour objet de rendre compte de l'ensemble de ce travail qui, au moment où est rédigé le présent texte, fait l'objet d'une thèse en cours, en ergonomie. Il s'agit ici de présenter les résultats du volet quantitatif de cette recherche.

\section{Une approche statistique dans un groupe aéronautique}

7 L'établissement qui constituait le terrain de recherche appartient à un grand groupe industriel français, dans lequel la médecine du travail s'est dotée d'un observatoire quantitatif pluriannuel qui fonctionne depuis 2002: le dispositif Evrest (Évolutions et relations en santé au travail), qui a depuis été décliné au niveau national et interentreprises (Molinié et Leroyer, 2011). Cet observatoire a été construit en collaboration par des médecins du travail et des chercheurs, avec pour objectif de pouvoir analyser et suivre dans le temps différents aspects du travail et de la santé des salariés. Il a été conçu pour durer, et pour cela il est forcément plus léger et "généraliste » qu'une enquête ponctuelle sur un problème particulier. Il s'agit d'un dispositif ancré dans la pratique des médecins du travail et porté par eux.

8 Sa genèse dans cette entreprise visait à répondre à deux objectifs principaux : produire des « indicateurs » à la fois sur le travail et sur la santé, et sur leur évolution, mais aussi pouvoir faire écho aux préoccupations de l'ergonomie de l'activité.

Cet observatoire Evrest s'appuie donc sur un questionnaire court (un recto-verso) ${ }^{1}$ rempli par un salarié au cours de chaque visite médicale systématique (ces visites sont espacées de un à deux ans dans cette entreprise, selon le métier des salariés). Ce questionnaire comporte, outre les données administratives du salarié, des questions sur ses conditions de travail (horaires, contraintes de temps, appréciation sur le travail, charge physique), déplacements professionnels, formation, mode de vie, et une dernière partie remplie par le médecin avec des informations assez générales sur l'état de santé du salarié (notamment cardiorespiratoire, neuropsychique et, ce qui nous importe ici, ostéoarticulaire).

Les problèmes de TMS étaient une préoccupation importante des médecins du travail de certains établissements du groupe. Ce qu'ils constataient dans leur activité clinique a été appuyé par des résultats quantitatifs issus d'Evrest (Buchmann et coll., 2009), puisque plus généralement dans l'entreprise les prévalences des problèmes ostéo-articulaires sont élevées, pratiquement du même ordre qu'au niveau national, alors que pour tous les autres problèmes de santé la situation dans cette entreprise semble nettement meilleure qu'en moyenne nationale. C'est chez les personnels d'atelier que d'une part la prévalence est la plus élevée, et d'autre part qu'elle augmente le plus avec l'âge. En regardant ce qui 
se passe du côté des conditions de travail selon l'âge, nous constations un léger phénomène de sélection mettant à l'abri les opérateurs les plus âgés, mais cette tendance très légère laissait penser qu'il n'y avait pas tellement de réaffectations possibles pour les opérateurs ${ }^{2}$ qui ont des problèmes ostéo-articulaires. Ces résultats chiffrés, suggérant des problèmes de TMS importants et stables dans le temps, constituaient un argument supplémentaire pour affirmer que les problèmes de santé ostéo-articulaires étaient à considérer prioritairement.

11 Dans cette recherche, Evrest a contribué à analyser de façon plus complète les relations entre le travail et la santé ostéo-articulaire au cours du temps, en cherchant à considérer l'état des douleurs, non seulement à un moment donné, mais dans un continuum, ce que l'on peut rapprocher d'une préoccupation plus générale : le débat social sur la santé au travail porte souvent sur des phénomènes qui méritent d'être analysés selon des approches diachroniques à moyen et long termes (Volkoff et Molinié, 2011). Car même s'ils sont a priori considérés comme relevant de faits instantanés, ils s'inscrivent dans la double histoire des systèmes de production et des parcours professionnels. La lecture diachronique des liens entre le travail et la santé, qui revêtent plusieurs formes, permet (avec les modèles et outils adaptés) de démêler les diverses dimensions temporelles impliquées dans ces phénomènes.

Compte tenu du thème de cette recherche et d'une entrée diachronique qui incorpore naturellement les questions d'âge, trois thèmes d'investigation seront successivement présentés en reprenant un découpage qui s'inspire des recherches sur le vieillissement. En effet, les textes en ergonomie abordant les phénomènes de vieillissement au travail suggèrent de distinguer trois processus : phénomènes d'usure (vieillissement «par » le travail), phénomènes de sélection (vieillissement "par rapport» au travail) et phénomènes de régulation (vieillissement «dans » le travail) (Volkoff et Gaudart, 2006). La présente recherche n'est pas centrée sur le vieillissement mais nous avons fait le choix de garder ce découpage, en substituant à l'analyse du vieillissement celle des troubles ostéo-articulaires, et en adoptant un ordre légèrement différent :

- Nous aborderons les régulations au travail dans un premier temps, justement parce que celles-ci, si elles sont efficientes, sont susceptibles de protéger de l'usure et de la sélection. Ainsi, les relations travail-santé seront tout d'abord analysées sous l'angle des processus de régulation, en essayant de déterminer si à un moment donné de l'itinéraire professionnel certains aspects du travail des opérateurs permettent (ou non) de limiter les effets délétères connus des facteurs physiques sur les douleurs.

- Puis nous aborderons les phénomènes d'usure et de cumul car ils renvoient aux traces du passé, quand la régulation n'est pas suffisamment protectrice. La santé actuelle sera donc mise en relation avec des "parcours d'astreinte $"^{3}$ aux facteurs de risque TMS, afin de mettre en exergue des mécanismes d'usure.

- Nous traiterons dans un troisième temps les mécanismes de sélection ou mise à l'abri liés aux troubles articulaires, à l'œuvre généralement lorsque les régulations ont échoué ou demeurent insuffisantes, et que l'usure devient trop importante pour tenir dans son travail. Cette troisième partie sera donc consacrée à l'analyse de ces mécanismes de sélection à l'œuvre au cours du temps, en considérant les variations dans le temps des astreintes ${ }^{4}$ physiques, en lien avec l'état de santé dans un stade initial.

13 La batterie de données Evrest à disposition a été utilisée avec des techniques différentes et sur des populations distinctes selon le thème investigué. Pour faciliter la lecture, nous indiquerons pour chacune de ces trois approches, à quel cadre conceptuel plus particulier 
nous nous référons, quelles analyses nous avons réalisées (méthodes et modes de calculs utilisés, à partir de l'outil commun déjà présenté : l'observatoire Evrest), quels résultats nous obtenons, et quels commentaires ces résultats suscitent à notre avis. Une discussion plus générale regroupant les trois approches sera présentée en fin d'article.

\section{Les mécanismes de « régulation »: les marges de manœuvre permettent-elles de limiter la survenue des douleurs, ou de mieux leur faire face?}

\subsection{Rappels conceptuels}

Pour introduire ici l'idée de régulation nous pouvons nous référer à Vézina (2001) : «La personne par son activité devra s'adapter et s'ajuster continuellement. Ainsi, elle développera différentes façons de faire ou stratégies qui lui permettront de tenir compte de la variabilité des conditions de travail et de sa propre variabilité afin de maintenir un équilibre entre sa santé et sa production. Lorsque ce processus de régulation est mis en échec, la santé peut être affectée ".

Par conséquent, des marges de manœuvre réduites peuvent conduire au développement de TMS. Il existe donc différentes modalités de régulations que les opérateurs construisent et mettent en œuvre pour répondre aux exigences de la tâche malgré leurs douleurs articulaires. Laville et Volkoff (2004) en proposent une catégorisation selon laquelle deux principales modalités de régulation sont reconnues, la régulation par évitement (qui peut être individuelle ou par répartition de tâches dans un collectif) et la régulation par compensation, qui se traduit par la mise en place de régulations fonctionnelles, possibles uniquement si l'organisation et les moyens de travail laissent des marges de manœuvre individuelles et collectives suffisantes (marges temporelles notamment).

\subsection{Méthode d'analyse}

Ce volet d'analyses repose sur l'étude des 3453 opérateurs ayant rempli une fiche Evrest en 2009. Les facteurs de risque TMS sont d'abord cernés par la question « Votre poste de travail présente-t-il des difficultés particulières sur les plans suivants : posture / gestes répétitifs / efforts, charges lourdes ? ", avec pour modalités de réponse à chacun des trois types de contrainte physique "oui, importantes », «oui, modérées », ou "non, pas du tout $»$.

17 Compte tenu du nombre limité de questions dans la fiche Evrest, on ne peut évidemment pas proposer une évaluation précise de ces régulations potentielles (ou de leur absence). Pour s'en approcher cependant, les variables utilisées ici vont être les suivantes (figure 1). 
Figure 1. Variables de régulation potentielle face aux facteurs de risque TMS

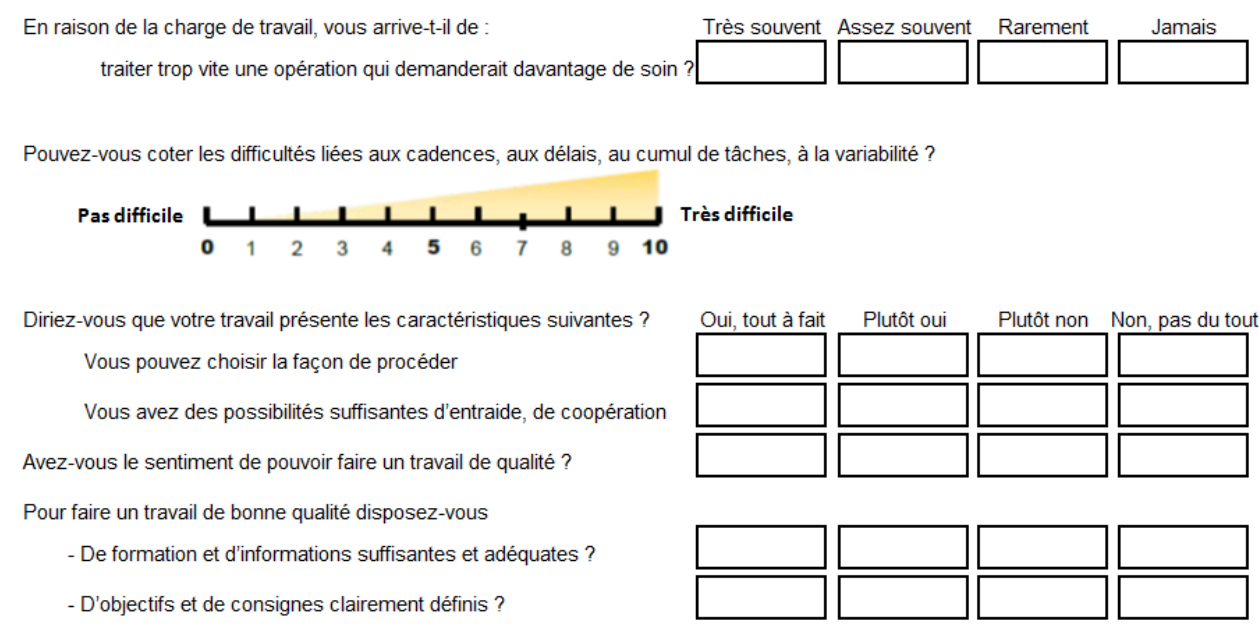

18 On voit que certaines régulations potentielles interrogent directement les marges de manœuvre temporelles, alors que les autres se centrent davantage sur la capacité d'initiative, les coopérations possibles, ou plus généralement les moyens d'assurer la qualité du travail.

Pour explorer le volet "régulation" dans le travail de ces opérateurs en étudiant les associations à l'œuvre, on regarde par le biais d'une régression logistique les liens entre un problème de santé ostéo-articulaire (variable à expliquer) et des variables explicatives : d'une part les facteurs de risque TMS et l'âge, d'autre part les variables de régulation potentielle choisies. Toutes choses égales par ailleurs, on veut voir si les variables de régulation sont susceptibles de " protéger » des problèmes ostéo-articulaires, à côté de l'âge et des contraintes physiques qui en sont des «facteurs aggravants ». On présuppose ici un sens causal qui n'est pas vérifié par la régression elle-même mais par des hypothèses issues de la littérature ergonomique que nous avons évoquée.

On peut, avec les données d'Evrest, aborder les questions de régulation également sous un autre angle. Les questions sur l'existence de problèmes de santé posées dans la fiche sont toutes accompagnées de l'interrogation: «Est-ce une gêne dans le travail ?» (réponse «oui » ou « non »). Il est donc possible d'étudier également les éventuelles régulations face aux gênes dans le travail, pour les opérateurs atteints d'un problème de santé. Pour cela, on décale le modèle précédent: le fait d'être gêné dans le travail par un problème ostéo-articulaire est la variable à expliquer, et on se place non plus au niveau de l'ensemble des opérateurs, mais au niveau de ceux qui sont concernés par ce problème de santé. En 2009, 976 opérateurs présentent des problèmes au niveau des vertèbres, 538 au niveau des membres supérieurs.

\subsection{Résultats}

21 Lorsqu'on applique le modèle qui «explique » les problèmes au niveau des vertèbres, on vérifie d'abord les effets aggravants de l'âge et des expositions physiques. Mais certaines variables semblent bien jouer un rôle de régulation (ou protecteur, selon le vocable consacré en épidémiologie), puisqu'elles s'accompagnent d'un odds-ratio (OR) inférieur à 1 (figure 2) : les marges de temps dans l'action (« ne pas - «plutôt non » ou « non pas du 
tout» - traiter trop vite une opération qui demanderait davantage de soin»), les possibilités de s'organiser («avoir - « oui tout à fait » ou " plutôt oui » - le choix de la façon de procéder »), et la clarté des objectifs assignés et des consignes reçues (en tendance seulement, car au seuil de 0,05 que nous avons retenu la significativité n'est pas parfaite). «Les possibilités de coopération » semblent en revanche avoir un effet plutôt aggravant.

Figure 2. Possibilités de régulations aux côtés des efforts physiques et de l'âge, vis-à-vis des problèmes de vertèbres (opérateurs 2009)

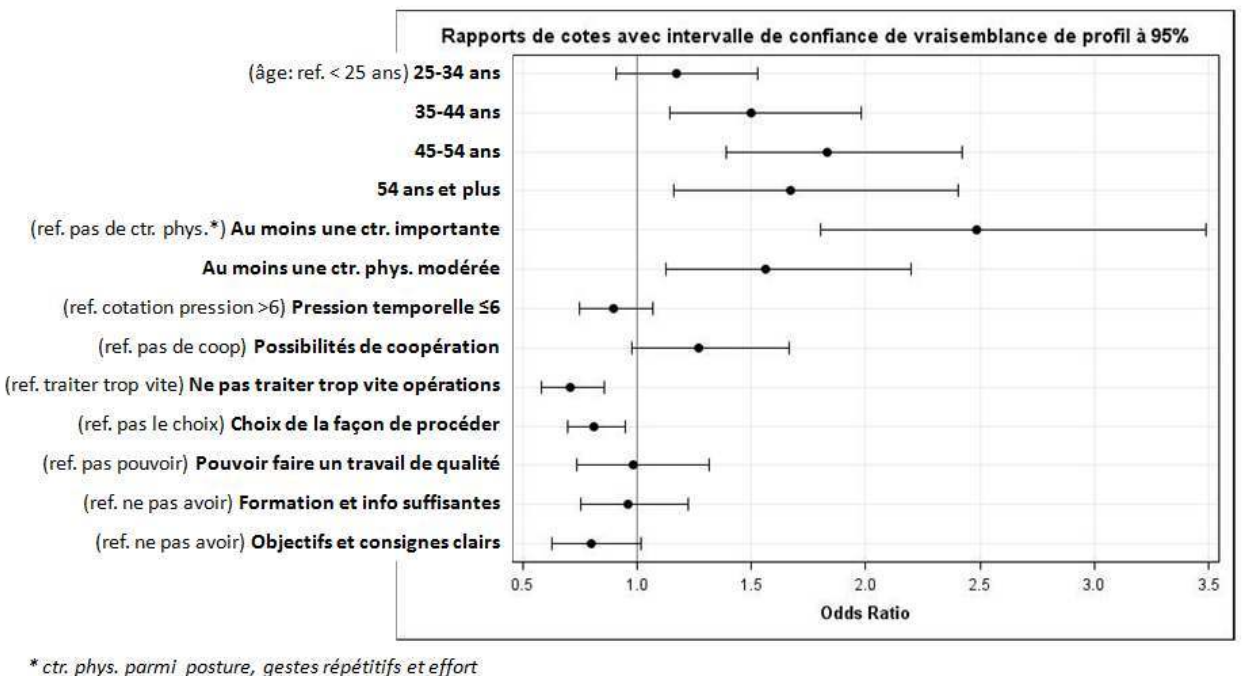

Par ailleurs, quand on reprend la même analyse en expliquant les problèmes de membres supérieurs, aucune variable ne ressort nettement comme moyen de régulation efficace (Buchmann, 2013). ${ }^{5}$

Lorsqu'on s'intéresse aux éventuelles régulations possibles vis-à-vis des gênes dans le travail (Buchmann, op. cit.), pour les opérateurs atteints de problèmes de vertèbres, «ne pas traiter trop vite une opération qui demanderait davantage de soin» ressort à nouveau comme variable « protectrice » $(\mathrm{OR}=0,7$, significativement différent de 1$)$, c'està-dire qu'elle diminue la probabilité d'être gêné dans le travail par ces problèmes de vertèbres, indépendamment de l'avancée en âge et des contraintes physiques qui, elles, augmentent cette probabilité. Pour les opérateurs affectés au niveau des membres supérieurs, c'est le sentiment de pouvoir faire un travail de qualité qui joue ce rôle de protection vis-à-vis des gênes dans le travail $(\mathrm{OR}=0,4)$.

\subsection{Discussion sur cette approche par les mécanismes de « régulation »}

$\mathrm{Au}$ vu de ces résultats, il existe donc dans certaines situations de travail de cette entreprise des possibilités de régulations des contraintes physiques vis-à-vis des problèmes ostéo-articulaires, passant par divers aspects de l'organisation du travail. Il existe aussi (même si elles sont moins nombreuses) des régulations possibles vis-à-vis des gênes dans le travail pour ceux déjà concernés par un problème ostéo-articulaire.

Par ailleurs, les membres supérieurs et les vertèbres ne sont pas à considérer de manière équivalente vis-à-vis de ces mécanismes de régulation. 
L'effet aggravant du manque de possibilité de coopération sur la probabilité d'avoir des problèmes de vertèbres n'est pas entièrement une surprise dans ce type de production, où le travail en coopération s'accompagne souvent de contraintes pour que chacun s'adapte aux gestes et rythmes des collègues (Millanvoye et Colombel, 1996).

27 Quant au rôle protecteur du «travail de qualité » vis-à-vis des gênes dans le travail dues aux douleurs du membre supérieur, elles peuvent renvoyer à une conception adéquate des outils et matériaux, qui permettent à la fois d'atteindre des objectifs de qualité (très élevés, dans la construction aéronautique, pour des raisons de coûts des produits, et de sécurité des aéronefs fabriqués), et d'adopter des stratégies gestuelles qui protègent l'organisme.

\section{Les mécanismes « d'usure ": quelles traces à moyen terme sur la santé des astreintes répétées ou prolongées?}

\subsection{Rappels conceptuels}

28 On l'a dit, lorsque les possibilités de régulation sont insuffisamment protectrices, notamment parce que l'organisation et les moyens de travail ne laissent pas les marges de manœuvre individuelles et collectives suffisantes pour les déployer, il y a usure des opérateurs au travail (Krause et coll., 2004). Considérer le cumul des «astreintes» au cours du temps est une manière d'étudier les phénomènes d'usure éventuellement à l'œuvre dans l'entreprise.

Les choix faits ici pour définir « l'astreinte » s'appuient sur la littérature sur les facteurs de risque TMS (Harkness et coll., 2003; Ijzelenberg et coll., 2004), et en particulier le modèle des TMS de Buchmann et Landry (2010b), qui identifie différents facteurs de risque TMS: des facteurs biomécaniques, des facteurs psychosociaux, et des facteurs mécaniques.

Dans cette recherche, nous avons trouvé utile de distinguer les notions de contrainte et d'astreinte. En nous appuyant sur des pistes proposées par Molinié (2003) ou Falzon (2004), dans une vision de l'opérateur actif dans son environnement, nous abordons l'astreinte comme une combinaison des contraintes de la situation de travail et des manières dont celles-ci sont perçues, vécues ou régulées par l'opérateur ou le collectif.

\subsection{Méthode d'analyse}

31 Dans la logique de ce qui précède, on peut intégrer le manque de possibilités de régulations à la définition même de la population « astreinte ».

Pour ce faire, une variable d'astreinte aux facteurs de risque TMS a été construite, à partir de plusieurs questions d'Evrest : trois questions sur la charge physique du poste de travail (à savoir les difficultés dues aux postures, aux gestes répétitifs, et aux efforts ou port de charges lourdes), mais aussi la question sur la possibilité de choisir la façon de procéder, et une cotation - de 0 à 10 - des difficultés dues à la pression temporelle, à partir de 
l'échelle analogique utilisée dans la fiche. On considérera qu'un opérateur est «astreint aux facteurs de risque TMS » dans deux cas :

- s'il a répondu «oui, importante» à l'une des trois questions sur la charge physique de travail, auquel cas cette seule réponse suffit pour juger qu'il y a un facteur de risque ;

- ou bien s'il a répondu " oui, modérées » à l'une au moins de ces questions tout en ayant coté à au moins 7 sur 10 les difficultés liées à la pression temporelle, et déclaré ne pas avoir le choix de la façon de procéder (réponse «plutôt non » ou «non pas du tout »); il s'agit donc alors de considérer que la charge physique n'est pas en soi un facteur de risque certain, mais que ses conséquences sont aggravées par l'obligation de se dépêcher et le manque de marges de manœuvre.

En cohérence avec le modèle précité, la variable d'astreinte construite s'appuie sur des facteurs biomécaniques, traduits ici par les trois questions sur la charge physique du poste de travail, et sur des facteurs psychosociaux, avec la question sur le choix de la façon de procéder et avec l'échelle analogique de cotation des difficultés dues à la pression temporelle. En revanche, aucun facteur mécanique (pression, chocs, vibrations) ne figure dans le questionnaire d'Evrest, mais ils ne manquent guère ici puisque les analyses d'activité réalisées sur sites ou encore les documents de prévention des risques ne relèvent que des expositions très marginales à ces facteurs.

Pour mettre en relation la santé ostéo-articulaire actuelle et l'astreinte au cours du temps, nous avons créé une variable de "séquence d'astreinte» des individus, et considéré les liens entre ce parcours et leur santé actuelle.

Dans ce but, nous avons limité ici l'étude à la sous-population des opérateurs vus au moins trois fois, sur un laps de temps le plus long possible, pour mettre en relation des " séquences d'astreinte » et leur lien avec la santé. Il s'agit de regarder ce qu'il advient d'un individu, selon qu'il est astreint ou non astreint à trois dates successives. La date t3 correspond à une fiche Evrest remplie en 2008 ou 2009 (pour ceux qui ont été vus les deux années, la fiche la plus récente est prise en compte). Symétriquement la date $\mathrm{t} 1$ correspond à l'année 2002 ou 2003, et cette fois c'est la fiche la plus ancienne qui est conservée pour les opérateurs vus les deux années, l'idée étant de mettre un maximum de distance entre t1 et t3. La date t2 est médiane: il s'agit d'un Evrest rempli en 2005 ou 2006, en gardant la fiche la plus récente pour les individus vus les deux années. Une variable de « séquence d'astreinte » a été construite pour cette population qui rassemble 1117 opérateurs, avec 8 combinaisons possibles, allant des «astreints» aux trois dates aux « non astreints » aux trois dates.

D'une part, nous avons croisé ces séquences d'astreinte avec la santé des opérateurs à la date t3. D'autre part, nous avons inséré ces séquences d'astreinte comme variables explicatives ajustées sur l'âge de régressions logistiques expliquant les problèmes de vertèbres et de membres supérieurs.

Une deuxième manière d'aborder les mécanismes d'usure est d'examiner plus généralement le cumul des astreintes des opérateurs sur l'ensemble des années observées.

La population considérée ici est un peu plus vaste que la précédente, elle englobe tous les opérateurs présents en 2008-2009, soit 6141 individus qui ont rempli une fiche Evrest au moins l'une de ces deux années (en gardant ici aussi les données les plus récentes pour ceux qui auraient été vus à la fois en 2008 et en 2009). L'idée était de définir un indicateur de cumul d'astreinte au cours du temps pour cette population. Pour cela, il n'était pas 
possible de simplement compter le nombre de situations d'astreintes constatées au cours du temps, car ce nombre dépend du nombre de fois où les opérateurs ont rempli un Evrest, nombre variable d'une personne à une autre, selon la durée depuis laquelle elle est présente dans l'entreprise, la date d'entrée de son établissement dans le dispositif Evrest, la périodicité et le calendrier des visites médicales qu'elle a passées, etc. Pour surmonter cette difficulté la méthode qui a été adoptée est de balayer toutes les années de 2002 à 2009, en incrémentant l'indicateur de 1 chaque fois qu'il y a astreinte, et en retranchant 1 à l'indicateur chaque fois qu'il n'y a pas astreinte (figure 3). La valeur de cet indicateur varie donc selon les individus de -8 (pour ceux qui auraient été vus 8 fois sans jamais être astreints) à +8 (pour ceux qui auraient été vus les 8 années en étant astreints à chaque fois). La valeur 0 , par exemple, correspond aux opérateurs vus un nombre pair d'années, et qui ont été autant de fois astreints que non astreints.

Figure 3. Exemples de calcul de l'indicateur de cumul d'astreinte

\begin{tabular}{|c|c|c|c|c|c|c|c|c|}
\hline 2002 & 2003 & 2004 & 2005 & 2006 & 2007 & 2008 & 2009 & \\
\hline A & A & A & A & A & A & A & A & $=>$ cumul $=8$ \\
\hline$\underline{n A}$ & $\underline{n A}$ & $\underline{n A}$ & $\underline{n A}$ & $\underline{n A}$ & $\underline{n A}$ & $\underline{n A}$ & $\underline{n A}$ & $=>$ cumul $=-8$ \\
\hline A & $\underline{n A}$ & $x$ & A & $x$ & A & $\underline{n A}$ & $\underline{n A}$ & $=>$ cumul $=0$ \\
\hline$x$ & $x$ & $\underline{n A}$ & $\underline{n A}$ & $\underline{n A}$ & $x$ & $\underline{n A}$ & $\underline{n A}$ & $=>$ cumul $=-5$ \\
\hline$x$ & $x$ & $x$ & $x$ & $x$ & $x$ & $\underline{n A}$ & $\underline{n A}$ & $=>$ cumul $=-2$ \\
\hline A & $x$ & A & $n A$ & $n A$ & $\underline{n A}$ & $\underline{n A}$ & $x$ & $=>$ cumul $=-2$ \\
\hline A & $\underline{n A}$ & A & A & A & $\underline{n A}$ & A & A & $=>$ cumul $=4$ \\
\hline & & & & & \multicolumn{4}{|c|}{$\begin{array}{l}\text { Légende: } \\
\begin{array}{ll}\mathrm{A} & \text { astreint à la date considérée } \\
\frac{n A}{\mathrm{x}} \text { non astreint à la date considérée } & \text { pas d'Evrest rempli cette année-là }\end{array}\end{array}$} \\
\hline
\end{tabular}

Deux régressions logistiques ont été réalisées, l'une expliquant les problèmes de vertèbres, l'autre les problèmes au niveau des membres supérieurs, chez les opérateurs interrogés avec Evrest en 2008-2009. Le cumul d'astreinte, en cinq classes, figurait comme variable explicative, en contrôlant sur l'âge des opérateurs (en cinq classes également).

\subsection{Résultats}

La population "astreinte » représente à peu près un opérateur sur deux, avec des variations légères et irrégulières d'une année à l'autre; la prévalence des douleurs aux membres supérieurs ou aux vertèbres est à peu près stable elle aussi (de l'ordre de $20 \%$ et $30 \%$ des opérateurs respectivement). La séquence d'astreinte définie a été croisée avec la santé en $\mathrm{t} 3$, s'agissant des problèmes de vertèbres d'une part, et des problèmes de membres supérieurs d'autre part (figure 4).

On constate que les opérateurs pour lesquels la prévalence de problèmes de vertèbres est la plus importante (accompagnée d'un OR significativement différent de 1) sont ceux qui ont été astreints au moins deux fois sur trois, dont la dernière. De façon prévisible, les « trois fois non astreints » sont ceux qui présentent en proportion le moins de problèmes de vertèbres. 
Du côté des membres supérieurs, la plus forte prévalence touche, comme on pouvait le prévoir, les opérateurs trois fois astreints, et la plus faible, à nouveau les «trois fois non astreints ». Les non astreints au départ puis toujours astreints ensuite présentent aussi une prévalence élevée, comme c'était le cas pour les vertèbres. Les opérateurs astreints uniquement à la dernière date (séquence nA-nA-A) présentent aussi une prévalence notable de problèmes de membres supérieurs.

Figure 4. Problèmes de vertèbres et de membres supérieurs en $\mathrm{t} 3$, pour chaque séquence d'astreinte
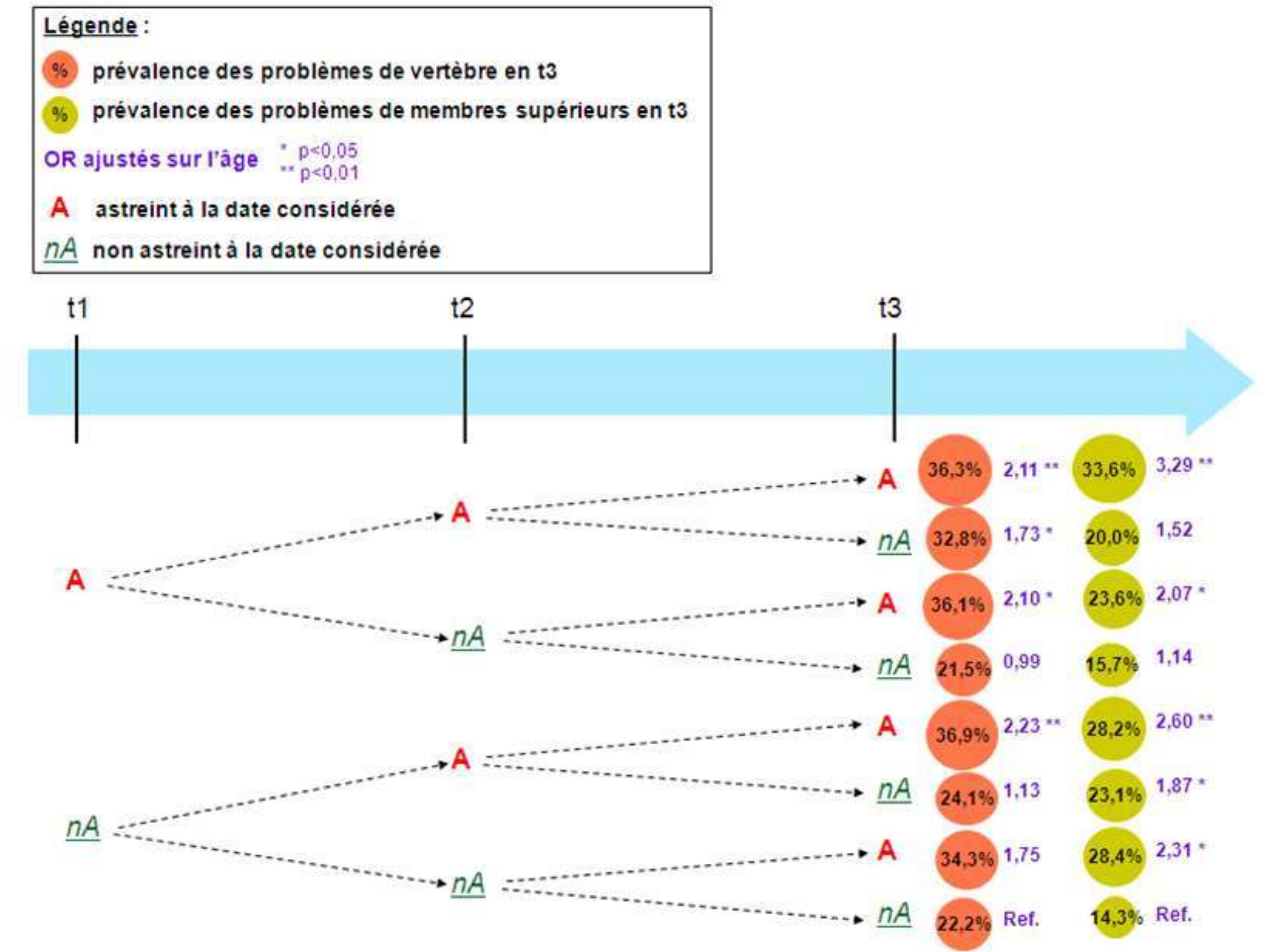

Parmi les opérateurs astreints aux trois dates (séquence $A-A-A), 36,3 \%$ présentent des problèmes de vertèbres en $\mathrm{t} 3$ (OR de 2,11 par rapport à la référence nA-nA-nA, indépendamment de l'âge). Parmi ces mêmes opérateurs, 33,6 \% présentent des problèmes de membres supérieurs en $\mathrm{t} 3$ (différence encore plus marquée avec la référence nA-nA-nA puisque I'OR vaut 3,29).

La figure 5 présente les résultats des régressions logistiques expliquant les problèmes de vertèbres et les problèmes au niveau des membres supérieurs par les valeurs du cumul d'astreinte, ajustées à l'âge. Les valeurs de cumul en deçà de -1 apparaissent comme "protectrices", notamment en ce qui concerne les problèmes de vertèbres (de façon significative puisque l'intervalle de confiance ne comprend pas la valeur 1). On observe donc que plus l'indicateur de cumul est bas, plus la probabilité d'occurrence de problèmes ostéo-articulaires est réduite, et à l'inverse plus le cumul est haut plus cette probabilité augmente. Donc plus on cumule d'astreintes au fil du temps, plus la probabilité d'avoir un problème de santé ostéo-articulaire est importante. 
Figure 5. Odds-ratios (et intervalles de confiance à $95 \%$ ) pour cinq classes de cumul d'astreinte, dans deux régressions logistiques expliquant d'une part les problèmes de vertèbres et d'autre part les problèmes de membres supérieurs, ajustés sur l'âge (en cinq classes), chez les opérateurs en 2008-2009.

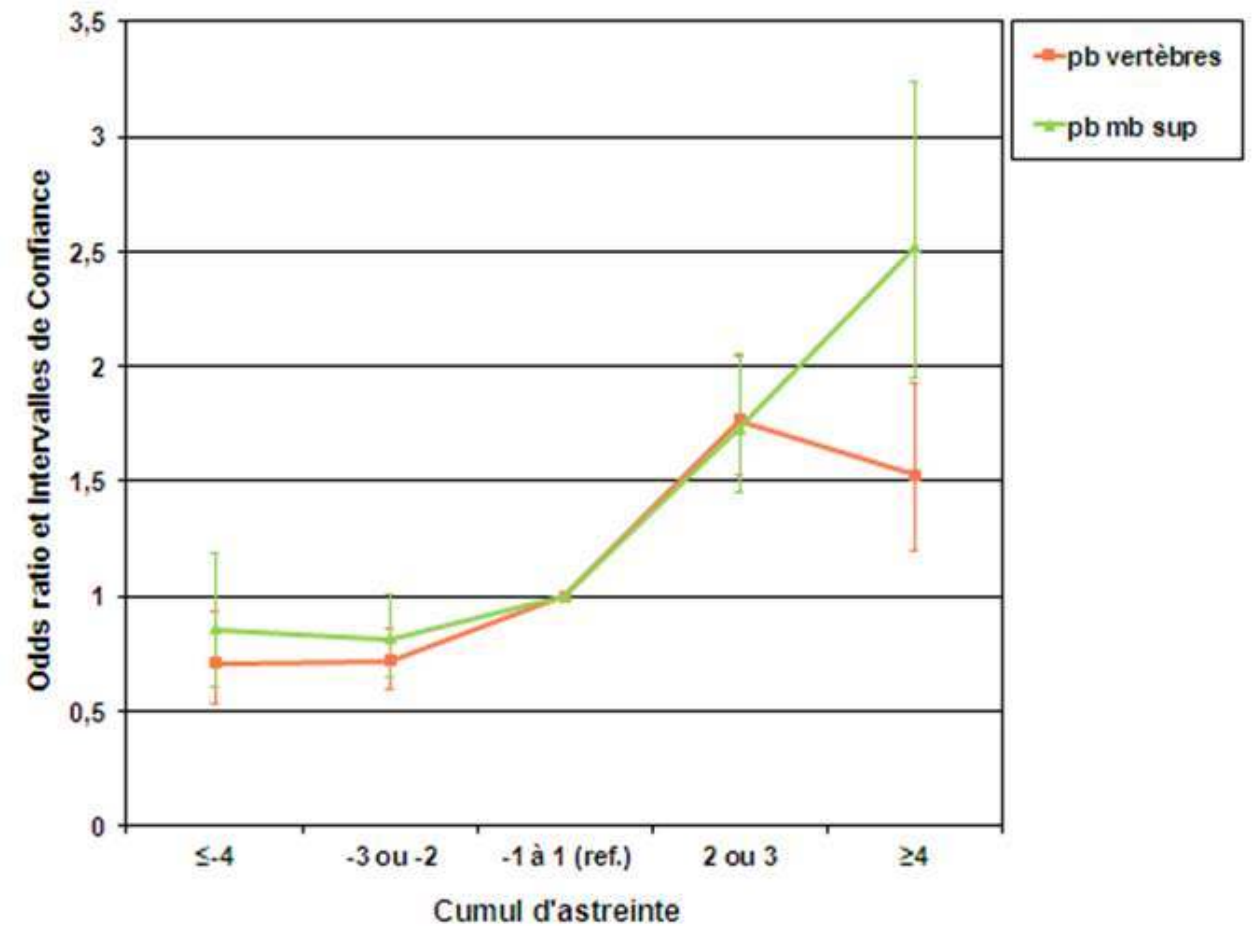

\subsection{Discussion sur cette approche par les mécanismes « d'usure »}

Le constat d'une absence de tendance claire au cours du temps de la prévalence de l'astreinte se fonde sur des comparaisons de la population étudiée, dans son ensemble, à différentes dates, sans voir si ce sont les mêmes personnes qui sont exposées ou non, malades ou non, d'une date à l'autre. Or, à chaque fois que des études longitudinales ont été réalisées à partir d'Evrest dans cette entreprise, on a constaté qu'entre une date $\mathrm{t} 1$ et une date $\mathrm{t} 2$ il y avait à la fois un bon nombre d'individus qui avaient moins de problèmes de conditions de travail, ou de santé, et un bon nombre pour qui la situation avait empiré dans l'un ou l'autre domaine, que l'évolution globale aille dans un sens ou dans l'autre, ou même quand il n'y ait globalement pas d'évolution entre les deux dates. Cela confirme l'intérêt de considérer aussi les relations travail/santé dans leur évolution à travers le temps en intégrant les évolutions individuelles.

La comparaison des pourcentages d'individus concernés en 2009 par des problèmes de vertèbres d'une part, de membres supérieurs d'autre part, au sein des populations astreintes et non astreintes nous a permis de constater (Buchmann, op. cit.) que le fait d'être sous astreinte actuellement est lié aux problèmes de santé, et que l'avoir été dans le passé, à quelque période que ce soit, est également lié à la santé actuelle. En revanche il n'y a pas de tendance claire qui apparaît selon la distance entre l'astreinte passée et la santé actuelle, c'est-à-dire que le lien entre la santé d'aujourd'hui et l'astreinte n'est pas plus ou moins fort selon que cette astreinte est plus ou moins éloignée dans le temps. Mais le lien entre trouble de santé et astreinte passée à une date quelconque, si on 
l'analyse par de simples tableaux croisés, peut provenir d'un autre lien statistique sousjacent : le fait d'être astreint à une date est évidemment corrélé avec le fait d'être astreint à une autre date (un opérateur astreint à un moment donné a plus de risque de l'être encore les années suivantes et de l'avoir été les années passées qu'un opérateur non astreint au même moment). Le lien apparent entre une astreinte passée et la santé actuelle pourrait donc n'être que le «reflet mathématique » du lien entre l'astreinte actuelle et la santé actuelle. Pour démêler cela, il était nécessaire de trouver des méthodes pour investiguer plus avant les relations dans le temps entre astreinte et santé, notamment en nous intéressant à ce que nous avons appelé la "séquence d'astreinte " des individus, et en considérant les liens entre cette séquence et leur santé actuelle.

Les constats réalisés en croisant la séquence d'astreinte et les problèmes de santé permettent de faire l'hypothèse que la répétition de l'astreinte dans le temps est un très important facteur de risque. C'est ce que confirme le fort gradient observé en croisant le cumul d'astreinte et la santé, ce qui renvoie bien sûr en premier lieu à une relation «dose/effet»: la répétition de l'astreinte indique une longue durée d'exposition. Une autre interprétation possible de ce résultat (qui peut être complémentaire à celle-ci) serait que la répétition de l'état "astreint " pourrait refléter en partie l'intensité de l'astreinte: si un opérateur est dans une situation d'astreinte forte et stable, elle sera réaffirmée d'une interrogation à l'autre, alors que si cette astreinte est stable mais plus modérée, les réponses de l'opérateur à Evrest pourront varier davantage d'une fois à l'autre, comme s'il marquait un peu d'hésitation au moment de répondre.

\section{Les mécanismes de " sélection »: les douleurs articulaires sont-elles à l'origine de réaffectations, à l'abri des astreintes?}

\subsection{Rappels conceptuels}

Cette partie de l'article relate les traitements statistiques produits en vue d'examiner les mécanismes de sélection à l'œuvre dans cette entreprise, en lien avec les facteurs de risque TMS. Dans cette optique, la santé est prise en compte comme facteur potentiel de réduction de l'astreinte, l'idée étant de montrer dans quelle mesure la santé passée peut, en raison d'un mécanisme relevant du " healthy worker effect ", s'avérer prédictrice des contraintes de travail actuelles, ou surtout de leur absence, par suite d'une «mise à l'abri » des travailleurs présentant des troubles de santé.

\subsection{Méthode d'analyse}

Nous avons observé les liens entre les douleurs articulaires chez les opérateurs astreints à une date et leur astreinte à une date ultérieure, dans deux sous-populations : d'une part les 1249 opérateurs vus en 2002 ou 2003 et vus également en 2008 ou 2009 (pour travailler sur un laps de temps assez large), et d'autre part les 2471 opérateurs vus en 2005 ou 2006 et vus également en 2008 ou 2009 (pour travailler sur un effectif plus important).

Nous avons également mené la même analyse en « durcissant » le critère de santé pris en compte : nous avons comparé les proportions d'opérateurs soustraits à l'astreinte entre 
deux dates selon qu'ils étaient sujets ou non, non seulement à des douleurs, mais à des limitations au niveau des vertèbres et des membres supérieurs.

Nous avons ensuite souhaité vérifier si les mécanismes de sélection seraient apparents en revenant aux items de base qui ont servi à construire la variable «d'astreinte». Nous avons donc cherché à voir si la santé à une date était prédictrice d'évolution des variables de charge physique au poste de travail composant notre variable d'astreinte, à savoir les difficultés en rapport avec la posture, les gestes répétitifs, et les efforts ou charges lourdes.

\subsection{Résultats}

51 La figure 6 présente le pourcentage d'opérateurs de chaque "séquence d'astreinte » (variable définie ci-avant), parmi les opérateurs astreints à la première date, selon qu'ils présentaient ou non un problème de santé à cette même date.

Figure 6. Séquence d'astreinte à venir selon la présence ou l'absence de problèmes de vertèbres et de membres supérieurs en $\mathrm{t} 1$, parmi les opérateurs astreints en $\mathrm{t} 1$ (le tableau contient des pourcentages colonne, la somme des colonnes est égale à 100).

\begin{tabular}{|c|c|c|c|c|}
\hline & $\begin{array}{l}\text { Opérateurs } \\
\text { astreints en t1 } \\
\text { avec } \\
\text { problèmes de } \\
\text { vertèbres }\end{array}$ & $\begin{array}{l}\text { Opérateurs } \\
\text { astreints en t1 } \\
\text { sans } \\
\text { problèmes de } \\
\text { vertèbres }\end{array}$ & $\begin{array}{l}\text { Opérateurs } \\
\text { astreints en t1 } \\
\text { avec } \\
\text { problèmes de } \\
\text { mb sup }\end{array}$ & $\begin{array}{l}\text { Opérateurs } \\
\text { astreints en t1 } \\
\text { sans } \\
\text { problèmes de } \\
\text { mb sup }\end{array}$ \\
\hline$A \quad A$ & 43,2 & 40,2 & 41,2 & 41,2 \\
\hline A A $n A$ & $23,8 \quad 67,0$ & $22,7 \quad 62,9$ & $26,8 \quad 68,0$ & $22,3 \quad 63,5$ \\
\hline A $n A$ A & 11,4 & 14,4 & 16,5 & 12,6 \\
\hline A $n A n A$ & $21,6 \quad 33,0$ & $22,7 \quad 37,1$ & $15,5 \quad 32,0$ & $23,9 \quad 36,5$ \\
\hline
\end{tabular}

43,2 \% des opérateurs astreints en $\mathrm{t} 1$ et qui avaient des problèmes de vertèbres à cette date ont été aussi astreints par la suite, en t2 et en t3. Si on s'intéresse seulement à l'astreinte en t1 et en t2, on voit que 67,0 \% des opérateurs astreints en 11 et qui avaient des problèmes de vertèbres à cette date étaient aussi astreints en $\mathrm{t} 2$.

On observe des différences assez peu importantes dans les répartitions des séquences d'astreinte entre les astreints avec problèmes de vertèbres en t1 (colonne 1) et les astreints sans problème de vertèbres en $t 1$ (colonne 2). Cependant, si l'on oppose les astreints en $\mathrm{t} 1$ et $\mathrm{t} 2$ aux astreint en $\mathrm{t} 1$ mais qui ne le sont plus en $\mathrm{t} 2$ (c'est-à-dire que l'on oppose les deux premières lignes aux deux suivantes), on constate qu'il y a un peu plus d'opérateurs astreints aux deux dates parmi ceux qui ont des problèmes de santé en $\mathrm{t} 1$, et qu'il y a donc parmi eux moins d'opérateurs mis à l'abri de l'astreinte entre t 1 et $\mathrm{t} 2$, qu'il s'agisse de problèmes au niveau des vertèbres ou des membres supérieurs. Par ailleurs, il $\mathrm{y}$ a bien moins d'opérateurs soustraits de l'astreinte dès $\mathrm{t} 2$ et en $\mathrm{t} 3$ parmi les astreints en $\mathrm{t} 1$ ayant des problèmes de membres supérieurs $(15,5 \%)$ que parmi ceux n'ayant pas ce type de problème de santé (23,9\%). Contrairement à ce que l'on aurait pu attendre ici, ces résultats ne montrent donc pas une sélection qui écarterait davantage des astreintes les opérateurs ayant un problème de santé en $\mathrm{t} 1$; ils reflètent plutôt un léger phénomène inverse. 
53 En resserrant l'analyse sur des comparaisons entre deux dates seulement, d'une part pour les 1249 opérateurs vus en 2002-2003 et en 2008-2009, et d'autre part sur les 2471 opérateurs vus en 2005-2006 et en 2008-2009, on constate dans les deux cas que les opérateurs soustraits de l'astreinte entre les deux dates ne sont pas spécialement plus nombreux en proportion parmi ceux présentant un problème de santé au départ (que ce soit au niveau des membres supérieurs ou des vertèbres), sans toutefois que l'on trouve cette fois de relation inverse bien établie.

54 En prenant en compte non pas les gênes dans le travail, mais les limitations occasionnées par les problèmes de santé considérés ici, nous avons observé ici un phénomène de sélection entre les deux dates en ce qui concerne les vertèbres (bien que statistiquement non significatif), pour les deux sous-populations considérées : 49,0\% des opérateurs avec limitations liées aux vertèbres en 2002-2003 étaient soustraits à l'astreinte en 2008-2009, alors qu'ils étaient seulement $43,2 \%$ chez les non limités (respectivement $40,7 \%$ et $36,2 \%$ si l'on restreint la distance entre les dates: pour la sous-population des opérateurs vus à la fois en 2005-2006 et en 2008-2009).

Sur la question de savoir si la santé à une date donnée est prédictrice d'évolution des variables de charge physique au poste de travail composant notre variable d'astreinte, un résultat en ce sens est bien trouvé dans les deux sous-populations d'opérateurs (différentes selon les dates retenues): avoir un problème au niveau des membres supérieurs parmi les exposés aux efforts ou charges lourdes à la première date est lié au fait de ne plus y être exposé à la seconde date : $80,9 \%$ des opérateurs avec un problème au niveau des membres supérieurs en 2002-2003 ont été soustraits à cette contrainte physique entre les deux dates, alors qu'ils étaient seulement $69,7 \%$ chez ceux ne présentant pas de problèmes à ce niveau, avec $\mathrm{p}=0,15$ pour le test du Chi-2 (respectivement $65,6 \%$ et $58,6 \%$ pour la sous-population des opérateurs vus à la fois en 2002-2003 et en 2008-2009, p =0,16). On n'observe cependant rien de tel à quelque niveau que ce soit pour les deux autres contraintes physiques explorées, ni par ailleurs pour les problèmes de vertèbres, quelle que soit la contrainte étudiée.

\subsection{Discussion sur cette approche par les mécanismes de « sélection »}

Dans l'ensemble donc, on n'observe plutôt pas ici de sélection au regard de «l'astreinte » telle qu'on l'a définie, sauf éventuellement en rapport avec les limitations du côté des vertèbres, et l'on constate qu'un phénomène de sélection semble opérer du côté des efforts et charges lourdes en rapport avec les problèmes au niveau des membres supérieurs.

57 On peut reprendre ici l'hypothèse, déjà proposée à propos de nos analyses de «l'usure ", qu'un opérateur astreint à une date et qui ne l'est plus ensuite (en prolongation sans doute d'un va-et-vient entre astreinte et non-astreinte antérieurement) l'est moins intensément qu'un opérateur qui apparaît astreint au deux dates (et peut-être astreint en continu auparavant), ces derniers étant de ce fait plus susceptibles de présenter des douleurs articulaires. Explorer cela relève d'un volet plus monographique d'analyse, mené par ailleurs au cours de cette recherche mais qui n'a pas vocation à être présenté dans le présent article. 


\section{Apports et limites d'une analyse statistique des douleurs articulaires dans l'entreprise}

Dans les entreprises - en France tout au moins - le seul décompte usuel des problèmes articulaires est le nombre de cas de maladies professionnelles (MP), reconnus par la Sécurité sociale, et concernant les affections des membres supérieurs ou du dos. Nous avons dit en introduction, et nous soulignons ici, que leur déclaration et leur reconnaissance relèvent d'une procédure délicate, au terme de laquelle seule une petite partie des affections dues au travail est effectivement évaluée. Dans l'entreprise étudiée ici, c'est manifeste: on l'a dit, la prévalence des douleurs aux vertèbres parmi les opérateurs de cette entreprise est voisine de $30 \%$, et de $20 \%$ pour les douleurs aux membres supérieurs, à rapporter à une population de l'ordre de 10000 opérateurs pour l'ensemble de l'entreprise - alors que le nombre de TMS reconnus, à une date donnée, dans cette entreprise est inférieur à 200 , donc environ $1 / 10$ de la population atteinte de douleurs.

59 L'ordre de grandeur des «TMS » reconnus, et des « douleurs articulaires », n'est donc pas du tout le même, et l'on peut admettre que ces dernières, outre le fait que leur grand nombre autorise des analyses quantitatives détaillées, constituent par elles-mêmes un problème légitime de santé au travail. C'est en tout cas l'optique que nous avons adoptée dans cette recherche, et l'importance des liens entre les caractéristiques du travail et la survenue de ces troubles nous y encourage : pour rester sur les résultats de la figure 3 , on peut retenir qu'une exposition prolongée aux "astreintes", telles que nous les avons définies (au moins une contrainte physique de niveau important, ou au moins une de niveau plus modéré mais assortie de contraintes organisationnelles), s'accompagne de probabilités fortement accrues que se développent des troubles vertébraux (OR voisin de 2 , après ajustement à l'âge) ou aux membres supérieurs (OR pouvant atteindre 3,7) - alors que toute cette analyse se situe au sein même de la population des « opérateurs ", c'est-àdire des personnels d'atelier, dont aucun n'est vraiment exempt de sollicitations physiques.

60 La troisième partie de notre analyse, portant sur la "sélection", accentue les préoccupations que nous venons d'évoquer. Elle montre en effet que, dans ce milieu professionnel aux métiers précisément définis, il n'y a guère de mobilité protectrice pour les opérateurs, nombreux, atteints de douleurs articulaires; en tout cas, rien de très spécifique : des mouvements de situations « astreintes » à « non astreintes » ont bien lieu, surtout pour les opérateurs en deuxième partie de carrière (comme nous avons pu le constater par ailleurs), mais ils ne prennent que rarement la forme de réaffectations ciblées pour raison de santé. Nos observations plus monographiques sur le terrain l'ont d'ailleurs confirmé: dans un établissement de quelques centaines d'opérateurs, en reprenant avec le médecin du travail les dossiers d'opérateurs qui auraient été changés de poste de travail en raison de leurs douleurs articulaires, nous avons pu rassembler neuf cas en tout, sur une période de plusieurs années.

$61 \mathrm{Au}$ demeurant, les réaffectations ne constituent, comme on l'a dit, qu'un pis-aller, puisqu'elles sont porteuses de risques accrus pour les opérateurs encore en bonne santé, dès lors surexposés. La diminution du nombre d'opérateurs «astreints » reste donc un objectif essentiel, surtout dans un contexte de vieillissement de la population. Rappelons ici que cette catégorie des « astreints » est définie par nous, eu égard aux connaissances 
disponibles en ergonomie surtout, à partir d'une combinaison entre exigences physiques (efforts, postures, répétitions) et restriction des marges de manœuvre dans l'activité de travail (pression du temps, manque de marges de manœuvre). S'agissant des efforts physiques, des possibilités d'amélioration ont déjà été largement utilisées dans cette entreprise; de nouveaux progrès sont certainement possibles, mais dans le secteur de la construction aéronautique un engagement important du corps au travail est difficilement évitable. La première partie de notre analyse statistique vient ici rappeler qu'une action sur les volets organisationnels, un certain desserrement des contraintes de temps, un accroissement des possibilités de choisir sa façon de procéder, une appréciation plus fiable des objectifs de travail constituent aussi des voies de prévention qui retiennent l'attention : une situation "positive » dans ces trois domaines à la fois (rappelons qu'en pareil cas les OR se multiplient) est assortie d'un OR global de 0,44 pour la présence de douleurs de vertèbres, donc une division par deux environ de leur prévalence, l'âge et le niveau de contraintes physiques étant fixés. De ce point de vue, un examen vigilant des réorganisations en cours dans ce secteur, et notamment des méthodes relevant du Lean Management (Buchmann et coll., 2010a), est particulièrement nécessaire.

En reprenant les quelques conclusions que l'on vient de présenter, on peut considérer que ces approches quantitatives sont assez probantes, puisqu'elles permettent à la fois d'attirer l'attention sur des facteurs d'atteintes à la santé, d'en nuancer l'analyse (tous les liens ne sont pas de même ampleur, ni toujours dans le sens attendu), et d'inscrire la démarche dans une perspective de moyen et long termes. Cependant, la tonalité prudente des interprétations que nous avons proposées reflète notre souci de ne pas considérer que ces chiffres "parlent d'eux-mêmes ». Pour comprendre de façon précise les conditions dans lesquelles les régulations sont développées (ou les limites auxquelles elles se heurtent), apprécier les raisons pour lesquelles un opérateur a pu fournir des réponses amenant à le classer comme " astreint » (ou non) à telle ou telle période de son parcours, analyser les réaffectations - quand elles sont possibles - et leurs conséquences individuelles et collectives, il faut s'efforcer d'articuler les résultats chiffrés avec des analyses d'activité, et des entretiens comportant une dimension rétrospective. C'est le but visé par cette recherche dans son ensemble.

\section{BIBLIOGRAPHIE}

Aptel, M., Vézina, N. (2008). Quels modèles pour comprendre et prévenir les TMS ? Pour une approche holistique et dynamique. Dans Actes $d u 2^{e}$ Congrès francophone sur les TMS, Montréal, Canada, 1-27.

Askenazy, P., Cartron, D., De Coninck, F. et Gollac, M. (2006). Organisation et intensité du travail. Toulouse, Octarès Editions.

Aublet-Cuvelier, A., Aptel, M., Weber, H. (2006). The dynamic course of musculoskeletal disorders in an assembly line factory. International Archives of Occupational and Environmental Health, 79, 7, 578-584. 
Berg, M., Sanden, A., Torell, G. et coll. (1988). Persistance of musculoskeletal symptoms: a longitudinal study. Ergonomics. 31, 1281-1285.

Buchmann ,W., Bellies, L., Volkoff, S. (2010a). What possibilities for sustainable prevention of WMSD within lean manufacturing production methods? Proceedings of the $7^{\text {th }}$ international Scientific Conference on Prevention of MusculoSkeletal Disorders, PREMUS, Angers, France.

Buchmann, W., Landry A. (2010b). Intervenir sur les TMS. Un modèle des troubles musculosquelettiques comme objet intermédiaire entre ergonomes et acteurs de l'entreprise? @ctivités, 7, 2, 84-103.

Buchmann, W., Mardon, C., Archambault, C., Volkoff, S. (2009). Combining occupational health data and ergonomics to take early action on Musculoskeletal Disorders. Proceedings of the $17^{\text {th }}$ Congress of the International Ergonomics Association (IEA), August 9-14, Beijing, China.

Buchmann, W., Volkoff, S., Archambault, C. (2011). L'approche diachronique des TMS, une paire de lunettes pour l'ergonomie myope ? Dans F. Jeffroy et A. Garrigou (Dir.), L'ergonomie à la croisée des risques, $46^{\mathrm{e}}$ Congrès de la Société d'ergonomie de langue française (Self) Comment l'ergonomie peut-elle contribuer à une gestion intégrée des risques ?, 14-16 septembre, Issy-les-Moulineaux, France

Buchmann, W. (2013). Aspects de moyen et long termes dans la genèse et l'évolution des troubles musculo-squelettiques au travail. Une recherche dans l'industrie aéronautique. Thèse de doctorat en ergonomie, CNAM.

Cole, D.C., Manno, M., Beaton, D., Swift, M. (2002).Transitions in self reported musculoskeletal pain and interference with activities among newspaper workers. J Occup Rehabil, 12(3), 163-174.

Diricq, N. (2011). Rapport de la commission des accidents du travail et des maladies professionnelles instituée par l'article L. 176-2 du Code de la sécurité sociale. (Rapport Diricq). Les TMS en Europe (2007). Définitions et données statistiques. (Rapport Eurogip 25F). www.eurogip.fr Falzon, P. (dir.) (2004). Ergonomie. Paris : PUF.

Fauconnier, D., Pépin, M., Douillet, Ph. (2005). Approche économique de la problématique des troubles musculo-squelettiques. Des coûts aux risques stratégiques. Dans Colloque DARES - ANACT. Paris, France.

Ha, C., Roquelaure, Y. (2010). Troubles musculo-squelettiques d'origine professionnelle en France. Où en est-on aujourd'hui ? BEH numéro thématique TMS, p. 35-37.

Harkness, E.F., Macfarlane, G.J., Nahit, E.S., Silman, A.J., McBeth, J. (2003). Mechanical and psychosocial factors predict new onset shoulder pain: a prospective cohort study of newly employed workers. Occup Environ Med., 60, 850-7.

Ijzelenberg, W., Molenaar, D., Burdorf, A. (2004). Different risk factors for musculoskeletal complaints and musculoskeletal sickness absence. Scandinavian Journal of Work, Environment \& Health. 30, 56-63.

Krause, N., Rugulies, R., Ragland, D., Syme, S.L. (2004). Physical workload, ergonomic problems, and incidence of low back injury: a 7,5-year prospective study of San Francisco transit operators. Am J Indust Med, 46, 570-85.

Laville, A., Volkoff, S. (2004). Vieillissement et travail. Dans P. Falzon (Dir.), Traité d'ergonomie. Paris, France: PUF. 
Mergler, D. (1999). Combining quantitative and qualitative approaches in occupational health for a better understanding of the impact of work-related disorders. Scandinavian Journal of Work, Environment \& Health, 15 (4), p. 54-60.

Millanvoye, M., Colombel, J. (1996). Âge et activité des opérateurs dans une entreprise de construction aéronautique. Dans R. Patesson (s/d), Intervenir par l'ergonomie - Actes du XXXI ${ }^{\mathrm{e}}$ Congrès de la Société d'ergonomie de langue française. Bruxelles (2, 39-46).

Molinié, A.-F. (2003). Interroger les salariés sur leur passé professionnel : le sens des discordances. Revue d'épidémiologie et de santé publique, 51, 589-605.

Molinié, A.-F., Leroyer, A. (2011). Suivre les évolutions du travail et de la santé : EVREST, un dispositif commun pour les usages diversifiés. PISTES, 13 (2). http://pistes.revues.org/1852

Plouvier, S., Gourmelen, J., Chastang, J.F., Lanoë, J.L., Leclerc, A. (2011). Low back pain around retirement age and physical occupational exposure during working life. BMC Public Health, 28;11:268.

Silverstein, B., Viikari-Juntura, E., Fan, Z.J., Bonauto, D.K., Bao, S., Smith, C. (2006). Natural course of nontraumatic rotator cuff tendinitis and shoulder symptoms in a working population. Scand $J$ Work Environ Health, 32, 2, 99-108.

Vézina, N. (2001). La pratique de l'ergonomie face aux TMS : ouverture à l'interdisciplinarité. Dans Comptes rendus du $36^{e}$ Congrès de la Société d'ergonomie de langue française et du $32^{e}$ Congrès de l'Association canadienne d'ergonomie. Montréal, Canada, 44-60.

Volkoff, S., Gaudart C. (2006). Vieillissement et travail. Actualités et dossier en santé publique, 57, 52-54.

Volkoff, S., Molinié A.-F. (2011). L'écheveau des liens santé travail, et le fil de l'âge. Dans A. Degenne, C. Marry et S. Moulin (Dir.), Les catégories sociales et leurs frontières. Laval, Québec : Presses de l'Université Laval.

Volkoff ,S. (2012). Dérives et inerties dans la démographie de la population salariée. Dans Molinié A.-F., Gaudart C., Pueyo V. (Dir.), La vie professionnelle : âge, expérience et santé à l'épreuve des conditions de travail. Toulouse : Octarès, Coll. « Travail et activité humaine », janvier, p. 21-30.

\section{ANNEXES}

Le questionnaire du dispositif Evrest du groupe aéronautique étudié (version 2009) 
CONDITIONS DE TRAVAIL.

I - Depuis la dernière vikite avez-vous changé de travall ?

Si a pour rasuns medcaies ?

II - Quels sont habituellement vos horxircy de travail ? Tpsplein

Oui

Normax $\square \quad 2 * 8 \square \quad 3 * 8 \square \quad$ VSD $\square \quad$ Autre
Non $\square$

Non $\square$

III - Contrainten de tempa I

- En redeun de la charge de travail, vous arrive l'il de

Depasser vos horaires normaxx Sauter ou ecourter un repas, ne pas prencre de pause Trabser tog vibu unc optration qui dsmanderat. dowantage de soin

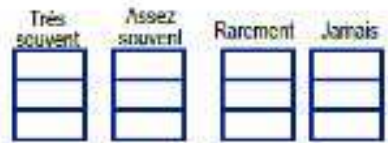

- Pouvez vous coter les difficultés hees aux cadences, aux délaus, au cumul de taches, a la variabahté

Pas difficile
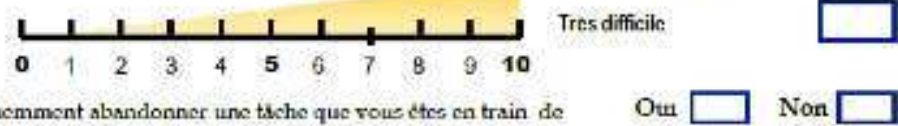

- Devoz voue frequemment abandonner une tache que vous ctos en train de feare puru une talse nom previve?

- Se crus dernez-woass que catta interruption d 'nctivita :

(1 seule réponse)

Perturte volre trovsi

Est sans consciquences poù vedre trowai

Fst un axpect pasilf de veline linusil

IV - Appriciations sur le travail : Diriez-veus que volue travuil prêyente les canuclérishagues suivealey ?

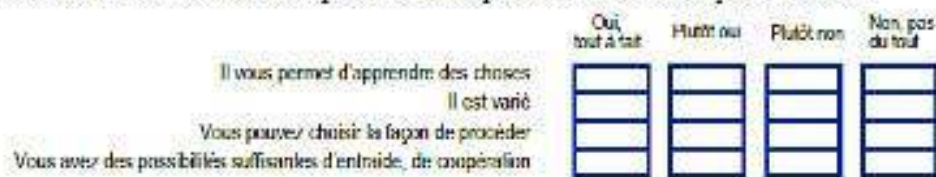

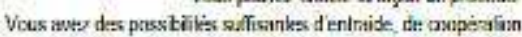

Avez vous le sentimuent que votre traval est recuma par votre enteturape profacsionnel ?

f:tes-vocis ph train de vivra ou vous attondez-vous $\mathrm{s}$ un changement non desere dars votra situsation de trasail ?

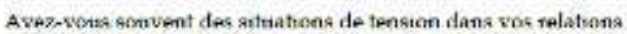
avec la hierarchic, bes colle gues, les clients ....?

Avez vous le sontiment de pouvoir taire un travail de qualits ?

Pouse faina un travail da bonne qualite disposaz- vous:

De formation ct d informations sextisantes ct askquales 0 objectrs et oce cons grnes cijeremart detrics

\begin{tabular}{|c|c|c|c|}
\hline v-Charge physique du poste de travail & $\begin{array}{l}\text { Qui } \\
\text { imporantes }\end{array}$ & $\begin{array}{l}\text { Oi. } \\
\text { modkrees }\end{array}$ & $\begin{array}{l}\text { Nen,pas } \\
\text { dulkul }\end{array}$ \\
\hline Votre posite de travail presente I'at & & & \\
\hline 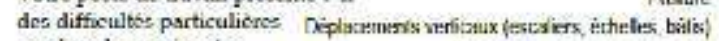 & & & \\
\hline Imporarts ceplecestents a pied & & & \\
\hline Gesles repélils & & & \\
\hline Flints itargess iunntes & & & \\
\hline Slsiun debout proinngic & & & \\
\hline
\end{tabular}


VII Avez-vous des déplacements professionaels:

Ou $\square \operatorname{Non} \square$

Si oui : Moine d'1 foie/semains $\square \quad 1$ fois/senains $\square$

Avez-vous eu des missions hors CEE depuis la dernière vieite?

Trouvez-vous ces đéplacements diffiolles ?

Plus de 2 fois/ semaine

Ou $\square$ Nor

ou $\square$ Nix

FORMATION

t-Depuis la dernière visite avez - vous eu use formation?

Oui

Non $\square$

Si oui, ctait ce une formation vulre prote de travail ? $\square$ un fulur poset?

en rapport aves

II - Avex-vaus eu depuis la derničre visite un rôle de formeteur, de tutorat ? ila $\square$ Non $\square$

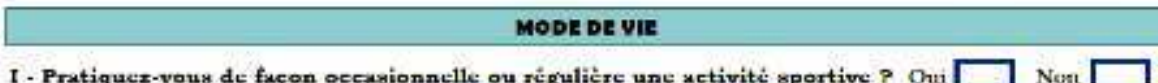

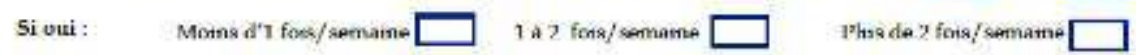

II - Consommations usuetles dont le week End

\begin{tabular}{|c|c|c|c|c|c|c|}
\hline & - & 1 & 2 & 3 & & \multirow{3}{*}{$\begin{array}{l}\text { Oui } \\
\text { Non }\end{array}$} \\
\hline Tabac en ciparetien/y & 0 & Mous des & sa 15 & Pins de 15 & \multirow{3}{*}{ Ex fameur } & \\
\hline 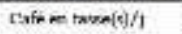 & 0 & 1 & $2 \times 1$ & Phan inat & & \\
\hline Alcool enveres / $/$ & 0 & Molue de 2 & 224 & Pae des & & \\
\hline
\end{tabular}

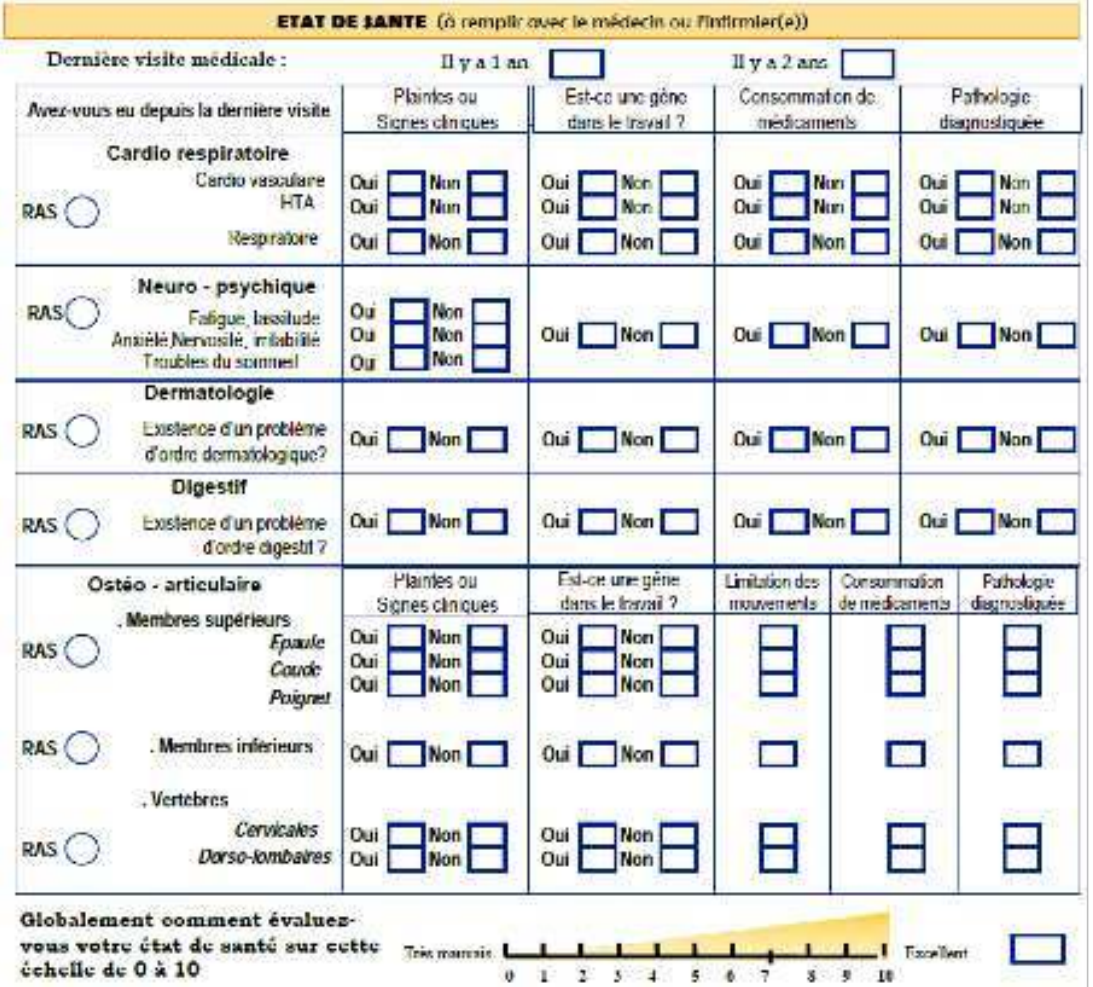

\section{NOTES}

1. Ce questionnaire est présenté dans son intégralité en annexe.

2. Dans la suite de cette article, le terme «opérateur » désignera les personnels d'atelier, allant de l'agent de fabrication au chef de ligne par exemple, et en opposition avec les professions administratives et RH.

3. Nous définirons alors ce terme.

4. Voir note précédente. 
5. Pour limiter la longueur du présent article, certains résultats de la recherche ne peuvent être présentés intégralement, même s'il est important de les évoquer. En pareil cas nous nous référerons à la thèse elle-même.

\section{RÉSUMÉS}

Cet article se base sur la partie quantitative d'une recherche en ergonomie dont le but est de comprendre comment des évolutions du travail ont favorisé ou limité la survenue et la persistance de troubles musculo-squelettiques (TMS) chez des opérateurs d'un groupe industriel aéronautique, avec une approche diachronique des faits étudiés. Un observatoire, fondé sur le recueil systématique de données quantitatives auprès des opérateurs (dispositif Evrest), permet de mettre en relation les facteurs de risque TMS passés et présents de ces opérateurs avec leur santé ostéo-articulaire. Sur cette base, sont explorés les mécanismes de régulation, d'usure et de sélection éventuellement à l'œuvre. Pour ces deux derniers mécanismes, l'analyse repose sur l'étude de "séquences d'astreinte » et de "cumuls d'astreinte ", l'astreinte étant définie à partir d'une combinaison de questions sur les contraintes physiques, la pression temporelle et les possibilités de choisir la façon de procéder dans son travail.

This article is based on the quantitative part of an ergonomics study whose goal it was to understand how work modifications increased or limited the occurrence and persistence of musculoskeletal disorders (MSD) among operators in an aeronautical industrial group, using a diachronic approach to the empirical evidence. A monitoring system (Evrest), based on the systematic collection of quantitative data from the operators, allowed us to relate the operators' past and present MSD risk factors to their musculoskeletal health. Based on these relations, we explored possible worker strategies, wear and tear phenomena, and sheltering processes developed for workers suffering from serious joint diseases. With regard to the last two elements, the analysis was based on the study of "constraint sequences" and "constraint accumulation." The definition of constraint was based on a combination of questions about physical constraints, time pressure, and opportunities to choose how to do one's work.

Este artículo está basado en la parte cuantitativa de una investigación en ergonomía cuyo objetivo es comprender cómo evoluciones del trabajo favorecieron o limitaron la aparición y la persistencia de trastornos musculo-esqueléticos (TME) entre operadores de un grupo industrial aeronáutico, con un enfoque diacrónico de los hechos estudiados. Un observatorio, fundado en la recopilación sistemática de datos cuantitativos sobre los operadores (dispositivo Evrest), permite relacionar los factores de riesgo TME pasados y presentes de estos operadores con su salud osteoarticular. A partir de eso se exploran los mecanismos de regulación, de desgaste y de selección eventualmente en acción. Para estos dos últimos, el análisis se basa en el estudio de "secuencias de esfuerzo" y de "acumulación de esfuerzo", el "esfuerzo" siendo definido a partir de una combinación de preguntassobre las exigencias físicas, la presión temporal y las posibilidades de elegir la manera de proceder en el trabajo. 
INDEX

Mots-clés : troubles musculo-squelettiques, dispositif Evrest, régulation, usure, sélection Keywords : musculoskeletal disorders, Evrest device, strategies, wear, sheltering processes Palabras claves : trastornos musculo-esqueléticos, dispositivo Evrest, regulación, desgaste, selección.

\section{AUTEURS}

\section{CÉLINE MARDON}

Centre d'études de l'emploi, Centre de recherches et d'études sur l'âge et les populations au travail, Noisy-le-Grand, France.

\section{WILLY BUCHMANN}

Centre d'études de l'emploi, Centre de recherches et d'études sur l'âge et les populations au travail, Noisy-le-Grand, France.

\section{SERGE VOLKOFF}

Centre d'études de l'emploi, Centre de recherches et d'études sur l'âge et les populations au travail, Noisy-le-Grand, France. 